\title{
STUDENTS' HIGH ACHIEVEMENT \\ ON LEARNING STYLE PREFERENCES \\ IN CHINESE DEPARTMENT, BINUS UNIVERSITY
}

\author{
印尼建国大学中文系高学习成就学生学习风格之分析
}

\author{
Yetty Go \\ Chinese Department, Faculty of Humanities, Bina Nusantara University \\ Jln. Kemanggisan Ilir III No. 45, Kemanggisan - Palmerah, Jakarta 11480 \\ yetty@binus.edu
}

\begin{abstract}
Every student certainly demonstrates different achievement in her/his Chinese language learning process because every student has her/his own individual way to resolve their problems in learning. In learning process, student's individual differences exist. These differences lead to different learning speed and learning style of the student. The purpose of this study was to investigate the high achievement students'learning styles. This study was based on Reid's learning styles theory and also uses Reid's Perceptual Learning Style Preference Questionnaire (PLSPQ) to investigate student's learning styles. The main finding of this study is that student's learning style preference is group style. According to student learning style preferences results, students prefer to learn together with others or in group and learn in a more interactive way.
\end{abstract}

Keywords: student's high achievement, learning styles, learning strategies, group style

\section{内容摘要}

每个学生在学习中文的过程中, 展现的成就肯定有所不同, 因为每个学生都有他们自己采取的学习以 及解决学习困难的方式。学生在学习上都有个别差异所在, 这些个别差异使得学生展现出不同的学习效率和 学习风格。本研究旨在探讨印尼建国大学中文系高学习成就学生学习中文的学习风格。本研究的学习风格是以 Reid的学习风格论述, 并采用Reid的 “知觉学习风格偏好量表” (PLSPQ) 为本研究的研究工具。研究发现, 印尼建国大学中文系高学习成就学生倾向于小组风格为学习风格, 这表明学生比较喜欢与他人或者小组一起学 习, 以互助学习更佳。

关键词：高学习成就的学生，学习风格、学习成就、中文、小组风格 
前言

每个学生在学习中文的过程中, 展现的成就肯 定有所不同, 因为每个学生都有他们自己采取的学习 以及解决学习困难的方式。学生在学习上都存在着个 别差异, 除了在种族、文化、母语、性别等方面有所 差异以外, 其它也包括学生的学习能力、动机、兴 趣、态度等, 这些在学习上的个别差异也反映出学习 者在学习速率和学习风格的不同。

近年来出现不少有关学习者个别差异的研究, 有些研究中内容还进一步地探讨学习者的学习动机、 学习策略、学习风格等因素与学习成就的关系, 结果 表明这些因素对学生的学习成就会产生直接或者间接 的影响 (楊翼, 1998)。学生的学习风格偏好影响他们 在学习上的成就, 学生的学习风格越多, 学习成就就 越高。

本研究以印尼建国大学中文系的学生为研究对 象, 并采用 “知觉学习风格偏好量表” (PLSPQ) 进 行学习风格测试。希望本研究得出的结果可以了解高 学习成就学生们在学习上采用的学习风格, 能帮助教 师了解学生的学习情况以及找到有效的教学法, 这能 提高教学效率, 并提高学生的学习成就。

\section{研究方法}

研究对象

本研究的研究对象是232位印尼建国大学中文 系2009-2013学年度一年级至四年级学生中的2013年 下学期的综合课、听力课、口语课以及写作课成绩的 学期总分得 85-100分, 在印尼建国大学中文系该分数 范围是属于A级。高学习成就的印尼建国大学中文系 学生人数分配, 如下表:

表1高学习成就的学生人数分配

\begin{tabular}{ccc}
\hline 科目 & 人数 & 百分比 \\
\hline 综合课 & 79 & $34 \%$ \\
听力课 & 56 & $24.1 \%$ \\
口语课 & 95 & $40.9 \%$ \\
写作课 & 83 & $35.8 \%$ \\
\hline
\end{tabular}

研究方法

本研究以量表调查法, 调查印尼建国大学中文 系高学习成就学生的学习风格偏好类型。本研究采用 Reid的 “知觉学习风格偏好量表” (PLSPQ) 来测试学 生的学习风格, 并使用电脑统计软体SPSS 20进行资 料统计与分析。

本研究的学习成就是采用印尼建国大学中文系 2009-2013学年度一年级至四年级学生的2013年下学 期的学期总分为依据。本研究主要采用综合课、听力 课、口语课以及写作课的成绩, 成绩的总分包括期中 考试成绩、期末考试成绩以及平时成绩 (指教师在上 课给的小测验或功课的平均数)。

\section{研究工具}

Reid (1984) 的知觉学习风格偏好量表 (Perceptual Learning Style Preference Quesionnaire, PLSPQ) 主要通过 30 个陈述问题鉴定学生偏好以何种方 式吸收、处理和储存新的信息以及掌握新技能, 主要
以视觉风格 (visua1)、听觉风格 (auditory)、动觉 风格 (kinesthetic) 、触觉风格 (tactile) 、小组风 格 (group) 或者个人风格 (individual) 六种学习风格 (Reid, 1984)。

本量表形式为 5 等Likert量表， 1 代表「非 常不同意」，2代表「不同意」，3代表「没意见」 ，4代表「同意」，5「非常同意」，其分为六个部 分, 有视觉 (visual)、听觉 (auditory)、动觉 (kinesthetic)、触觉 (tactile)、小组 (group) 以及个人 (individua1)。每个部分共有5题，视觉部分由第6 题、10题、12题、24题和 29 题为代表, 听觉部分由第 1题、7题、9 题、 17 题和 20 题为代表, 动觉部分由第 2 题、8题、15题、19题和 26 题为代表, 触觉部分由第 11 题、14题、16题、22题和 25 题为代表, 小组部分由 第 3 题、 4 题、 5 题、 21 题和 23 题为代表, 个人部分由 第13题、18题、27题、28题和30题为代表。

笔者采用该量表的原因为通过该量表我们可以 了解学生在学习上使用什么风格来吸取、保留并学会 华语文。除此之外, Reid的知觉学习风格偏好量表涉 及面广、题量适中、已被广泛运用于语言领域的研究 中(Wintergerst, DeCapua \& Verna, 2003)。

\section{内容}

\section{学习风格的定义}

学习风格的概念, 是于1954年, 由美国学者 Herbert Thelen首次提出, 但是学习风格一词是直到 1970年前后才真正出现。根据Brown (2007) 所指出, 风格 (styles) 是指个人持续不变的倾向或偏好, 风格 是智能运作与人格种类的普遍特质, 介于情感与认知 之间。人的风格取决于自己内化整体世界的方式, 但 由于内化过程不完全和认知有关, 因此我们认为学习 风格中融合了生理、情意及认知领域。

Keefe 与Jenkins (1997) 认为学习风格是认 知、情意与生理行为三者表现的特征。此特征是学习 者对学习环境的感觉、互动及反应的一种相对稳定特 质。学习风格一般是用来鉴定学习者的学习及学习偏 好的方式。Dunn与Dunn (1993) 认为学习风格是生理 与发展个人特征的总合, 使同样的教学, 有可能对一 些学习者有效, 但对其他学习者无效, 因为每个学习 者都有不同的专注力、处理, 及保留新或较困难信息 的方式。学习风格是学习者吸取新信息使用的方式, 如使用整体型或分析型解决学习困难或倾向哪一种感 官方面学习 (0xford，2003)。

本研究认为学习风格是学生利用独特性且习惯 性的特质来吸收、处理与保留新信息或新技能的学习 方式, 其受到认知、情意等因素的影响。每个学生都 有自己独特的学习风格偏好, 而学习风格并没有好坏 的标准。学习风格立即影响到学习成就, 能了解自己 的学习风格的学习者, 能让自己的学习成就更佳 (游 政男, 2000)。

\section{Reid 的学习风格类风格}

Reid (1995) 认为每个学生都以不同的方式学 习, 学生的学习风格偏好可显示其以不同的情况下学 习更佳。Reid把学习风格分为六类风格, 即视觉风格 (visual)、听觉风格 (auditory)、动觉风格 (kinesthetic)、触觉风格 (tactile)、个人风格 (individua1) 及小组风格 (group)。Reid的六类学习风格, 详 细说明如下: 
1. 视觉风格: 以视觉为偏好的学习者以看书里、 黑板上及练习本里等从视觉刺激获取的信息学 习更佳。这些学习者以阅读新信息或指示, 记 忆并了解得更佳。学习者根本不需要通过很多 口语说明, 就可以自己一个人独立学习。

2. 听觉风格: 以听觉为偏好的学习者通过听他人 说话或解释可学习新信息。这些学习者以朗读 或动口说较容易记忆所学的新信息。学习者通 过听讲座、与他人会话和听口头指示等听觉输 入学习更佳。

3. 动觉风格: 以动觉为偏好的学习者较喜欢肢体 活动学习新信息。这些学习者参与活动、户外 教学, 及课堂上的扮演角色记忆信息更佳。

4. 触觉风格: 以触觉为偏好的学习者动手做事, 如在实验室做实验、动手拼图模型等触摸或亲 自动手完成学习任务更佳。写笔记或写指示也 可以帮学习者记忆更好, 以亲自参与有关活动 会帮他们了解新信息。

5. 个人风格: 以个人风格为偏好的学习者比较喜 欢单独读书。这些学生单独学习时, 学得比较 好。单独学习时, 学习者能够学会新信息及促 进学习过程。

6. 小组风格: 以小组风格为偏好的学习者, 偏爱 在小组里或者与他人一起完成学习任务, 一起 读书。通过小组合作可促进学习者的学习过程 与学会新信息。

本研究主要想了解印尼建国大学中文系学生是 比较倾向于哪种风格类型来吸取与保留信息，因此， 笔者根据Reid的学习风格论述作为探讨印尼建国大学 中文系学生的学习风格偏好类型框架。

探讨高学习成就学生的学习风格

学习者的学习风格可分很多种，有些人习惯一 个人单独学习, 对这种学习者来说, 其他人的存在可 影响他的学习过程, 也有些学习者如果跟其他人或者 群体一起学习时, 才可以学得更好 (Dunn, 1983)。 游政男 (2000) 指出能够了解适合自己学习风格的学习 者、能让学习成就更佳, 学习成就高的学生能够使用 许多学习策略来调整个人学习方式并解决学习困难 ( 吴越，2004）。

为了了解得高成就学生的学习风格偏好类型, 许多研究者对学习风格与学习者的中文学习成就进行 分析, 以下为高学习成就者的学习风格偏好之一些研 究:

\begin{tabular}{cll}
\hline 研究內容 & 研究者與年代 & \multicolumn{1}{c}{ 研究發現 } \\
\hline 高学习成就 & 彭妮絲 (2011) & 中高及中等阅读理解能力的学习风 \\
学生的学习 & & 格倾向于视觉风格。 \\
风格偏好 & 廖康伶 (2008) & $\begin{array}{l}\text { 字汇学习成就测验得高分的学习者 } \\
\text { 之风格类型是听弱视弱, 占被试者 } \\
\end{array}$ \\
& & \\
& & \\
& & 的 $50 \%$ 。 \\
\hline
\end{tabular}

学习风格并无熟优孰劣之分, 学生如能选择适 合个人的学习风格, 其能够快速地吸收新信息, 并能 够快速地掌握语言能力。

印尼建国大学中文系高学习成就学生学习风格偏好类 型
高综合成就的学生其学习风格偏好类型

高综合成就的学生, 小组风格 $(\mathrm{m}=2.795)$ 为其 第一高, 视觉风格 $(\mathrm{m}=2.570)$ 为其第二高, 个人风格 $(m=2.552)$ 为其第三高, 触觉风格 $(m=2.448)$ 为其第 四高, 动觉风格 $(m=2.408)$ 为其第五高, 最低者为听 觉风格 $(m=2.329)$ 。研究结果显示, 高综合成就的学 生倾向于小组风格为学习风格偏好类型。学生通过小 组讨论某语法点, 或一起完成综合练习, 能够提升他 们的综合课的成就。

表2 高综合成就的学生其学习风格偏好类型

\begin{tabular}{ccccc}
\hline 学习风格 & 总数 & 平均数 & 标准差 & 排名 \\
\hline 视觉风格 & 203.4 & 2.570 & .5511 & 2 \\
听觉风格 & 184.0 & 2.329 & .5044 & 6 \\
触觉风格 & 193.4 & 2.448 & .5166 & 4 \\
动觉风格 & 190.2 & 2.408 & .6222 & 5 \\
个人风格 & 201.6 & 2.552 & .6612 & 3 \\
小组风格 & 220.8 & 2.795 & .5809 & 1 \\
\hline
\end{tabular}

高听力成就的学生其学习风格偏好类型

高听力成就的学生, 小组风格 $(m=2.829)$ 为其 第一高, 视觉风格 $(\mathrm{m}=2.507)$ 为其第二高, 动觉风格 $(m=2.468)$ 为其第三高, 触觉风格 $(m=2.396)$ 为其第四 高, 个人风格 $(\mathrm{m}=2.296)$ 为其第五高, 而最低者为听 觉风格 $(\mathrm{m}=2.268)$ 。研究结果显示, 高听力成就的学 生倾向于小组风格为学习风格偏好类型。虽然学生不 是倾向于听觉风格, 但是听力成就还可以得高分, 表 示通过小组学习的方式, 也可以提升听力的能力。

表3 高听力成就的学生其学习风格偏好类型

\begin{tabular}{ccccc}
\hline 学习风格 & 总数 & 平均数 & 标准差 & 排名 \\
\hline 视觉风格 & 140.4 & 2.507 & .5173 & 2 \\
听觉风格 & 127.0 & 2.268 & .4336 & 6 \\
触觉风格 & 134.2 & 2.396 & .4756 & 4 \\
动觉风格 & 138.2 & 2.468 & .5673 & 3 \\
个人风格 & 128.6 & 2.296 & .6150 & 5 \\
小组风格 & 158.4 & 2.829 & .6260 & 1 \\
\hline
\end{tabular}

高口语成就的学生其学习风格偏好类型

高口语成就的学生, 小组风格 $(\mathrm{m}=2.743)$ 为其 第一高, 视觉风格 $(\mathrm{m}=2.602)$ 为其第二高, 个人风格 $(m=2.556)$ 为其第三高, 触觉风格 $(m=2.465)$ 为其第 四高, 听觉风格 $(m=2.356)$ 为其第五高, 而最低者为 动觉风格 $(\mathrm{m}=2.413)$ 。研究结果显示, 高口语成就的 学生倾向于小组风格为学习风格偏好类型, 这表明通 过小组方式学习, 学生更有机会运用华语文来讨论、 分享、交际等, 并提升学生的口语成就。

表4 高口语成就的学生其学习风格偏好类型

\begin{tabular}{ccccc}
\hline 学习风格 & 总数 & 平均数 & 标准差 & 排名 \\
\hline 视觉风格 & 247.2 & 2.602 & .5780 & 2 \\
听觉风格 & 223.8 & 2.356 & .5513 & 5 \\
触觉风格 & 234.2 & 2.465 & .6150 & 4 \\
动觉风格 & 229.2 & 2.413 & .6816 & 6 \\
个人风格 & 242.8 & 2.556 & .7277 & 3 \\
小组风格 & 260.6 & 2.743 & .5788 & 1 \\
\hline
\end{tabular}


高写作成就的学生其学习风格偏好类型

高写作成就的学生，小组风格 $(m=2.694)$ 为其 第一高, 视觉风格 $(m=2.547)$ 为其第二高, 个人风格 $(m=2.523)$ 为其第三高, 触觉风格 $(m=2.482)$ 为其第 四高, 动觉风格 $(m=2.463)$ 为其第五高, 而最低者为 听觉风格 $(m=2.337)$ 。研究结果显示, 高写作成就的 学生倾向于小组风格为学习风格偏好类型, 学生可以 在小组里面分享个人的经验, 多讨论能增加学生的华 语文字句，能够帮助增加写作文的技能。

表5 高写作成就的学生其学习风格偏好类型

\begin{tabular}{ccccc}
\hline 学习风格 & 总数 & 平均数 & 标准差 & 排名 \\
\hline 视觉风格 & 211.4 & 2.547 & .5301 & 2 \\
听觉风格 & 194.0 & 2.337 & .5084 & 6 \\
触觉风格 & 206.0 & 2.482 & .5562 & 4 \\
动觉风格 & 204.4 & 2.463 & .6112 & 5 \\
个人风格 & 209.4 & 2.523 & .6573 & 3 \\
小组风格 & 223.6 & 2.694 & .5458 & 1 \\
\hline
\end{tabular}

结语

本研究透过量表调查法, 针对232位印尼建国 大学中文系2009-2013学年度一年级至四年级高学习 成就学生进行学习风格测试, 研究结果显示高学习成 就印尼建国大学中文系综合课、听力课、口语课以及 写作课成就较高学生的学习风格偏好类型主要为小组 风格, 与视觉风格为主次风格。这表明学生与他人或 者与小组一起学习中文时, 亦通过视觉工具上课时, 学习得快并能提升学习成就。

根据研究得出的结果, 笔者建议针对倾向于小 组风格的学生, 教师在教学时可以使用分组讨论或者 比较活动性的教学法, 这很适合学生的学习风格, 并 可以改善倾向于个人风格的学生之学习模式, 并提升 他们的学习成就。若针对倾向于视觉风格的学生教师 可以多用图片、图表、图示或其他视觉工具并利用带 动作的学习, 其能够使视觉风格的学生表现的较好 ( 彭妮丝, 2011)。笔者也建议学生应该对自己的学习 风格加以了解, 其能够帮助自我学习达到较佳的效 果、吸收更快、保留更久。除此之外, 学生能够调整 并掌控自己的学习过程, 如此能够帮助学生提升自我 学习成就, 成为成功的学习者。

\section{参考文献}

廖康伶.（2008）. 国中生知觉学习风格, 字汇学习策 略与字汇学习成就之相关研究. 国立云林 技大 学技术及职业教育研究所硕士论文.

彭妮丝. (2011). 不同学习风格马来西亚海青生之 华语文阅读/教学策略. 中原华语文学报, 8 , $59-78$.

吴越.（2004）。大学生学习策略与场认知方式、学习 风格、学习动机以及学业成就关系的研究. 西 示范大学硕士论文.

杨翼.（1998）. 高级汉语学习的学习策略与学习效果 的的关系. 世界汉语教学，1，88-93.

游政男.（2000）。学习风格与超媒体网页架构方式 对学习钟摆周期之影响. 国立东华大学教育 究 所硕士论文.
Brown, H. D. (2007). 第二语教学最高指导原则 (译者: 林俊宏)。台北: 台湾培生教育.

Dunn, R. (1983). Learning Style and its Relationto Exceptionality at Both Endsof the Spectrum. Exceptional Children, 49, 496-506.

Dunn, K., \&Dunn, R. (1993). Teaching Secondary Students Through Their Individual Learning Styles: Practical Approaches for Grades 7-12. Massachusetts: A Pearson Education Company.

Keefe, J. W. and Jenkins, J. M. (1997). Instruction and the Learning Environment. NY: Eye on Education.

Oxford, R. L.(2003). LearningStyles and Strategies: An Overview. GALA, 1-25.

Reid, J. (1984). Perceptual Learning Styles Preference Questionnaire. In Reid, J. (Ed.), Learning Styles in the ESL/EFL Classroom. Boston: Heinle\&Heinle.

Reid, J. (Ed.). (1995). Learning Styles in the ESL/EFL Classroom. Boston:Heinle\&Heinle.

Wintergerst, A.C., DeCapua, A., and Verna, M. (2003). Conceptualizing Learning Style Modalities for ESL/EFL Students. System, 31, 85-106. 